\title{
The Radar Echo Telescope for Neutrinos
}

\author{
Krijn D. de Vries ${ }^{a, *}$ and Steven Prohira ${ }^{b}$ on behalf of the Radar Echo Telescope \\ Collaboration \\ (a complete list of authors can be found at the end of the proceedings) \\ ${ }^{a}$ Vrije Universiteit Brussel, IIHE-DNTK, Pleinlaan 2, Brussel, Belgium \\ ${ }^{b}$ Department of Physics, Center for Cosmology and AstroParticle Physics (CCAPP), The Ohio State \\ University, Columbus $\mathrm{OH}, \mathrm{USA}$ \\ E-mail: krijn.de.vries@vub.be, prohira.1@osu.edu
}

We present the Radar Echo Telescope for Neutrinos (RET-N). RET-N focuses on the detection of the cosmic neutrino flux at $>\mathrm{PeV}$ energies by means of the radar detection technique. This method aims to bridge the energy gap between the diffuse neutrino flux detected by IceCube up to a few $\mathrm{PeV}$ and the sought for cosmogenic neutrinos at $\mathrm{EeV}$ energies by the in-ice Askaryan detectors, as well as the air shower radio detectors. The radar echo method is based on the detection of the ionization trail in the wake of a high-energy neutrino-induced particle cascade in ice. This technique, recently validated in a beam test (T576 at SLAC) is also the basis for the RET-N pathfinder experiment, RET-CR, which is currently under development. Based on the T-576 results, we show that the radar echo method leads to very promising sensitivities to detect cosmic neutrinos in the PeV-EeV region and above. We present the RET-N project and the results of our sensitivity studies.

$37^{\text {th }}$ International Cosmic Ray Conference (ICRC 2021)

July 12th - 23rd, 2021

Online - Berlin, Germany

\footnotetext{
${ }^{*}$ Presenter
} 


\section{Introduction}

Neutrinos travel freely through space and are as such, if detected, the perfect messenger particle to probe the hadronic processes at the highest energy environments in our universe. The caveat is indeed found in its detection. As neutrinos are weakly interacting, their interaction cross-section is very low. Below PeV energies, Earth becomes transparent for neutrinos and as such very large volume detectors are needed to capture them. Moving to higher energies increases the interaction cross-section, but this is countered by the steeply falling flux of cosmic particles as observed for cosmic-rays at the highest energies. Although very hard to detect, the IceCube neutrino observatory in 2013 opened the field of neutrino astronomy by detecting the TeV-PeV cosmic neutrino flux [1, 2].

Recently the field of multi-messenger astronomy showed its value by the detection of a highenergy cosmic neutrino by IceCube. Directly after its detection, IceCube send an alert to a multitude of cosmic-ray, gamma-ray and neutrino detectors around the globe. This alert was followed up by these detectors, and a gamma-ray flare was observed within the reconstructed neutrino direction from the Blazar TXS-0506+056 [3, 4]. The chance coincidence of such an event to occur due to random background was rejected at the $3 \sigma$ level, providing the first possible high-energy neutrino source and showing the strength of multi-messenger astronomy.

With the first possible sources found through the multi-messenger channel, an interesting future lies ahead for particle astronomy. IceCube currently covers a $1 \mathrm{~km}^{3}$ detection volume, which allows to get neutrino events up to a few PeV before it runs low in sensitivity. Given that cosmic-rays up to $10^{20} \mathrm{eV}$ have been observed, and that neutrinos typically obtain $\mathrm{O}(\mathrm{few} \%)$ of the parent cosmic-ray after interacting, we do expect a neutrino flux up to $\mathrm{EeV}$ energies. To probe this flux, we need to instrument even larger volumes than the current $\mathrm{km}^{3}$ instrumented by IceCube. Several detectors have been proposed or are already operating in the search for the $>\mathrm{PeV}$ cosmic neutrino flux. Most of these detectors make use of the radio signal to probe such high-energy particle cascades. The advantage of the radio signal is primarily found in its long attenuation length, reaching up to kilometer distances in ice (depending on frequency). These detectors consist of in-ice Askaryan radio detectors that aim to detect the coherent Cherenkov emission in the radio-wave band from a neutrino-induced particle cascade [5-7], as well as balloon experiments like ANITA [8] that search for the same signals while hovering above the Antarctic ice sheet. A second type of detector searches for Earth skimming tau-neutrinos. In case the tau neutrino undergoes a charged-current interaction, a high-energy tau lepton is produced that is able to leave Earth and decay in-air to produce an air shower, from which either its radio signal is sought after [9], or the particle content can be sought for [10]. These methods, however, have a typical peak sensitivity close to $\mathrm{EeV}$ energies due to the coherent nature of the radio signal with its peak emitted radio power scaling roughly quadratic to the primary particle energy.

In this proceeding we discuss the Radar Echo Telescope for Neutrinos (RET-N) as a candidate detector to probe the $>\mathrm{PeV}$ cosmic neutrino flux. The radar echo method does not search for the coherent radio emission from the particle cascade itself (although in principle this signal could be observed), but rather it looks for the reflection of a transmitted radio wave off the ionization plasma left in the wake of a high-energy particle cascade. As the scattered signal amplitude scales directly to the transmitted signal power, this technique is very well suited to probe particle cascades down to $\mathrm{PeV}$ energies. Furthermore, as the signal properties are highly correlated to the emitted signal, 
a lot of flexibility is found for reconstruction of the primary neutrino properties (see proceeding "Simulation and Optimisation for the Radar Echo Telescope for Cosmic Rays" from this conference).

\section{From beam test to in-nature neutrino detection}

The radar echo technique to probe high-energy particle cascades was put forward already in the 1940's by Blacket and Lovell as a way to detect cosmic-ray-induced particle cascades in air [11]. These efforts were abandoned several years later once meteors were detected using this technique and attention shifted. Furthermore, doubt was cast upon the method's feasibility due to the suppression of the return signal by collisional damping effects. The method was revived again in the early 2000's by theoretical works [12] followed by experiment [13]. These efforts came to an end once limits were set on the effective scattering cross-section of an in-air particle cascade by the TARA collaboration, as well as theoretical advances, leading to the conclusion that the technique not feasible to probe air showers [14]. At the same time, however, the idea to use radar to probe in-ice particle cascades was put forward $[15,16]$.

For neutrino induced cascades in ice, owing to the increased density of the medium, the ionization density is greatly increased relative to cosmic ray cascades in air. Its application was immediately linked to the detection of the cosmic neutrino flux at the highest energies. As the proposed radio frequencies for the method have long attenuation lengths, the method is ideal to cover large volumes of ice and probe the cosmic-neutrino flux at the highest energies. Several experiments demonstrated the feasibility of in-medium radar scattering [15], and then attention was turned to lab-based experiments to detect particle-cascade induced ionization deposits. The first of these was performed at the Telescope Array Electron Light Source facility, where a relatively low energy $\mathrm{O}\left(\mathrm{PeV}\right.$ equivalent energy, $\left.40 \mathrm{MeV} / \mathrm{e}^{-}\right)$beam was directed in a block of Ice $[17,18]$. Although the data obtained during these experiments were inconclusive, it was used to gain insight into detector properties (non-linearities in our systems) and future analysis methods. It was the T-576 experiment performed at the Stanford Linear Accelerator Center (SLAC) [19, 20] that lead to the final breakthrough with the first ever detection of radio reflections from a particle cascade. The cascade was induced by directing a high-energy electron beam in a block of HDPE, having very similar properties to an in-ice induced particle cascade. Interestingly these results, showed effective radar scattering cross-sections of $\mathrm{O}\left(10^{-1} \mathrm{~m}^{2}\right)$, very well in agreement with RadioScatter simulations [21], as well as a full Finite Difference Time Domain simulation, including all relevant physics parameters such as collisional damping and free charge lifetimes.

Confirming our radar scattering model, the SLAC T-576 results lead to the formation of the Radar Echo Telescope collaboration. The goal of our collaboration is to instrument the Radar Echo Telescope for Neutrinos described in this work. We, however, do envision one step in between the SLAC T-576 experiment and the deployment of RET-N. This step is taken to show the in-situ feasibility of the method by means of the detection of cosmic-ray air showers penetrating a large altitude ice sheet. The air shower can be easily tagged from its particle content hitting the ice surface, which subsequently can trigger the in-ice radar system that searches for reflections of the in-ice propagation of the cosmic-ray particle cascade, allowing for in-situ verification of the method and the development of our radar system. To achieve these goals, we plan to deploy the Radar 

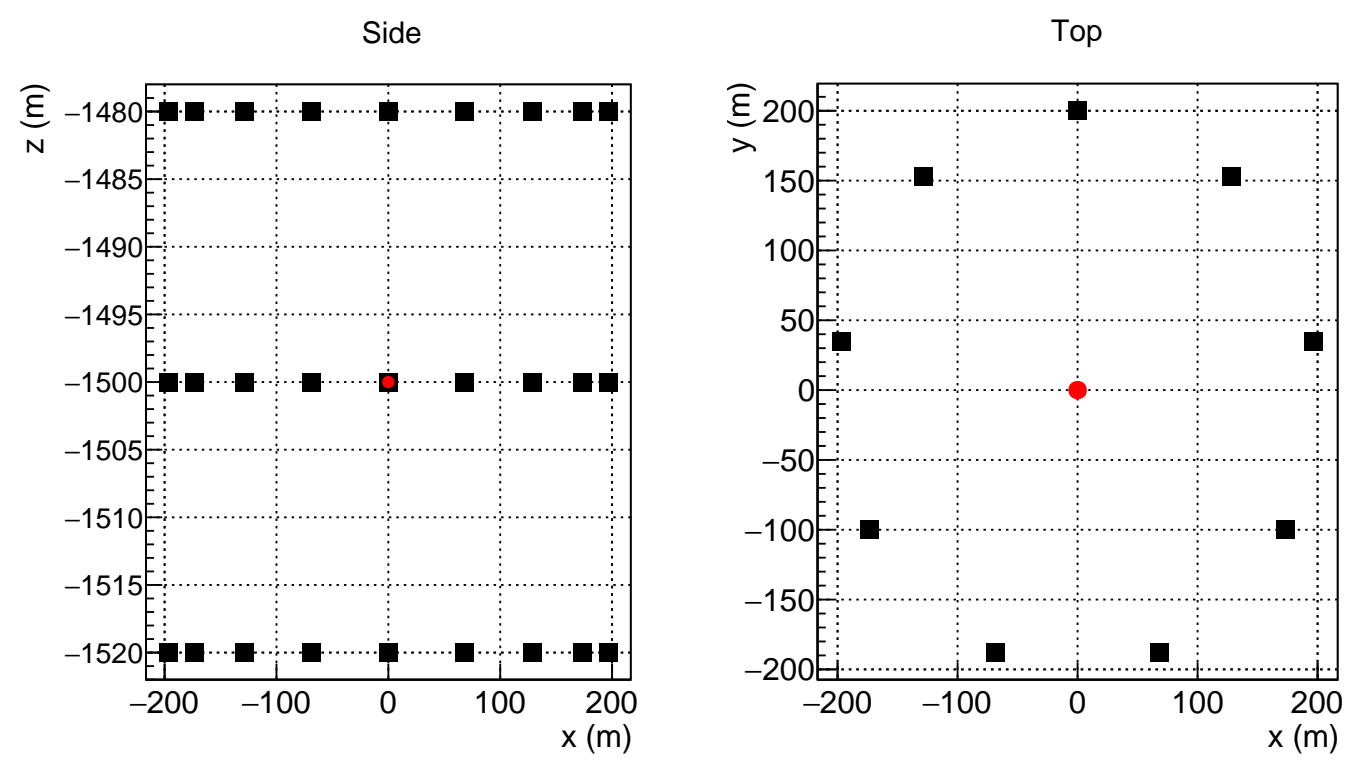

Figure 1: The layout of a single RET-N station consisting of a transmit antenna located at $1500 \mathrm{~m}$ depth emitting $40 \mathrm{~kW}$ of effective power, surrounded by 27 receiver antennas on a total of $9,200 \mathrm{~m}$ radial spokes with a vertical antenna spacing of $20 \mathrm{~m}$.

Echo Telescope for Cosmic Rays (RET-CR) in the near future. This experiment is currently under development; details can be found in [22].

\section{Detector layout and hardware}

Having our simulations confirmed by experiment allows us to calculate the sensitivity for a possible in-ice neutrino detector. Large sheets of ice for such a detector are found at South Pole, or Greenland. These ice layers are typically several kilometers of height, and are initially formed by snow accumulation which is compressed into ice. Due to this specific formation of the ice, the initial $\sim 100 \mathrm{~m}$ of ice consist of compressed snow with a strong density gradient. As is shown in [23], continuous radio wave propagation through this layer, the so-called firn, is highly non trivial and can lead to additional uncertainties in direction and energy reconstruction. As such, the proposed layout in this work is set well below the firn, in deep, uniform ice. It should, however, be noted that this is not the final proposed layout as this will depend on more detailed simulation studies on signal properties and reconstruction which are currently ongoing.

The detector configuration used for the sensitivities presented here is shown in Fig. 1, given by a central transmitter emitting a continuous wave of $250 \mathrm{MHz}$ at $40 \mathrm{~kW}$ of effective radiated power, similar to a typical FM radio broadcast station. It should be noted that this power is not necessarily emitted by a single isotropic antenna, but different configurations using a multiple antenna array layout can be envisioned (see for example [21]). In our simulation set-up we assume this power is emitted from from a central vertical dipole radiator at $1.5 \mathrm{~km}$ deep, $\vec{x}_{t}=(0,0,-1500) \mathrm{m}$, in the ice. As below $\sim 100 \mathrm{~m}$ the ice refractive index is constant at $n=1.78$, we ignore ray-tracing and ice irregularities. The central transmitter is surrounded by 27 receiver antennas on 9 strings that are 
located at $200 \mathrm{~m}$ radial distance from the transmitter and range from a depth of $1480 \mathrm{~m}$ to $1520 \mathrm{~m}$ with a vertical inter-antenna spacing of $z=20 \mathrm{~m}$.

\section{Sensitivity}

One large advantage for the radar detection method is the multitude of trigger options. As the received signal is a function of the transmitted signal, the expected signal can be tuned to have properties ideal for triggering. A direct example of this is found for the expected radar signal of a particle cascade probed by continuous wave emission. This signal contains is a distinct frequency shift over time (colloquially known as a 'chirp') when transmitting continuous wave RF emission due to the relative motion of the cascade with respect to the transmitter-receiver configuration. Already in this most basic configuration one can imagine, such a feature allows for triggering using a heterodyne trigger [24, 25].

The final sensitivity is obtained by generating a set of 500,000 events in a fiducial volume of $\sim 35 \mathrm{~km}^{3}$, given by a cylinder with a depth of $2800 \mathrm{~m}$ similar to the Antarctic ice layer, and a radius of $2 \mathrm{~km}$, surrounding the detector within the energy range of $10^{15}$ to $10^{20} \mathrm{eV}$. Earth absorption is taken into account by properly weighting the events by their zenith angle, with the azimuth of the event generated randomly. Furthermore, we consider the inelasticity distribution of neutral current events when simulating cascades in RadioScatter. The noise level is fixed at $5 \mu \mathrm{V}$ RMS, equal to thermal noise over a $50 \mathrm{MHz}$ bandwidth.

As shown in Fig. 2, it follows that 10 stations of the proposed detector (1 transmitter antenna, 27 receiver antennas, $40 \mathrm{~kW}$ effective power) provides excellent sensitivities in the $>\mathrm{PeV}$ energy range up to $\mathrm{EeV}$ energies and above. It should be noted that the sensitivity scales directly to the number of proposed detectors. Hence, increasing the number of stations to $\mathrm{O}(10)$ should allow to probe the highest energy neutrinos with excellent sensitivities using a relatively sparse detector layout covering a total area of hundreds of $\mathrm{km}^{2}$. One large advantage of the radar method is that this coverage is well below the areas that have to be instrumented by different Earth based detection methods probing the EeV neutrino flux. For example, the radar echo method has a large angular acceptance relative to Askaryan methods, which require that their detectors must lie within a band $\sim 1$ degree wide at the Cherenkov angle, as measured with respect to the cascade axis.

\section{Outlook}

In this proceeding we have presented the Radar Echo Telescope for Neutrinos (RET-N). With our simulation models confirmed by the SLAC T-576 experiment, we are able to calculate the sensitivity for a proposed detector layout in the deep ice, consisting out of a single transmitter with $40 \mathrm{~kW}$ effective transmit power, surrounded by 27 receiver antennas. These calculations show that due to its high trigger efficiency the radar echo method is able to probe the cosmic neutrino flux at the highest energies, while covering a limited surface area using sparse instrumentation.

The RET project consists out of two different phases. To confirm the radar method in-situ and develop clever triggering algorithms, we will first install the Radar Echo Telescope for Cosmic Rays (RET-CR). RET-CR aims to detect cosmic-ray particle cascades penetrating a large altitude 


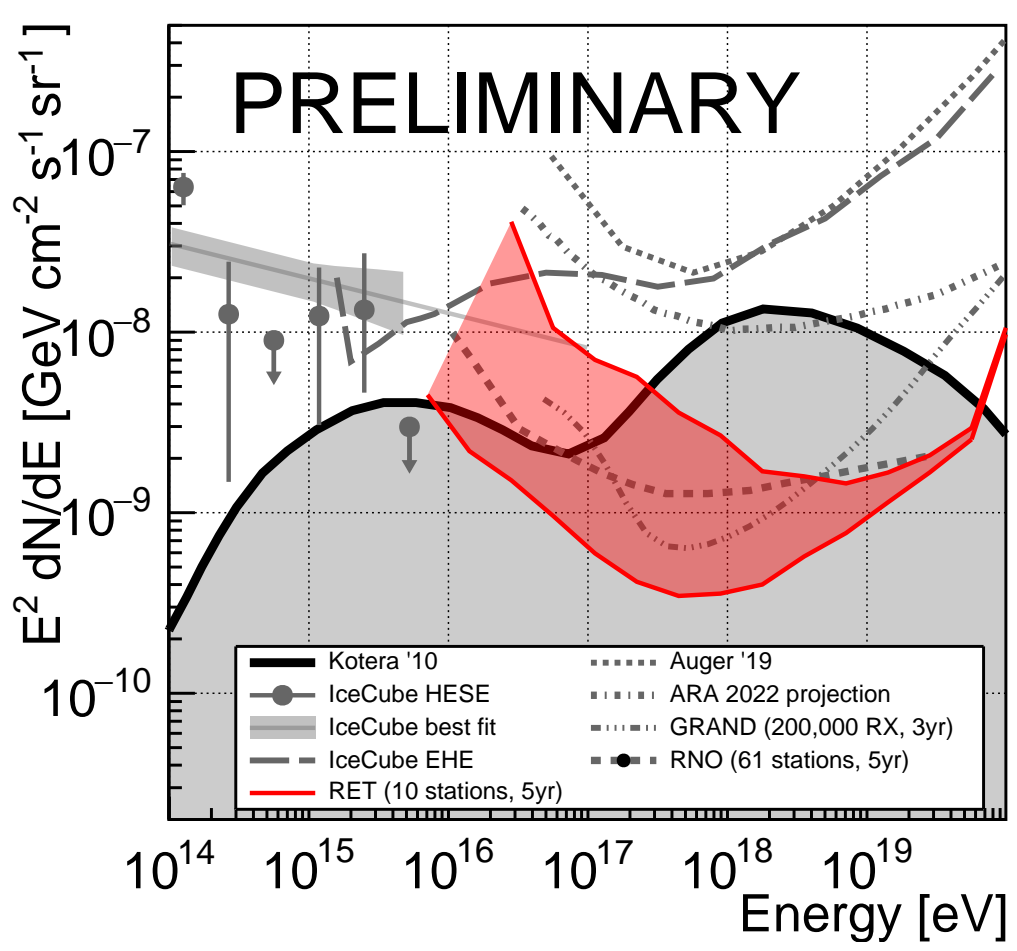

Figure 2: The RET-N neutrino sensitivity for 10 stations having the proposed layout of a single transmitter antenna emitting $40 \mathrm{~kW}$ effective power and 27 receiver antennas operating for 5 years. The red band shows triggering at different noise levels from $-6 \mathrm{~dB}$ to $+6 \mathrm{~dB}$, with the noise level at $50 \mathrm{MHz}$ bandwidth thermal noise.

ice sheet using an in-ice radar set-up that is triggered by a surface cosmic-ray detector. The RETCR development is currently in an advanced state with prototype stations already running (see Proceedings "Simulation and Optimisation for the Radar Echo Telescope for Cosmic Rays" and "Investigating signal properties of UHE particles using in-ice radar for the RET experiment" of this conference). Its on-site deployment is foreseen in the near future after prototyping has been completed.

The positive detection of air-shower cores by RET-CR and subsequent development of efficient trigger algorithms, will inform the final RET-N detector layout. We expect that RET-N will be deployed and detecting cosmogenic neutrinos within the decade.

\section{Acknowledgements}

RET-CR is supported by the National Science Foundation under award numbers NSF/PHY2012980 and NSF/PHY-2012989. This work is also supported by the Flemish Foundation for Scientific Research FWO-12ZD920N, the European Research Council under the EU-ropean Unions Horizon 2020 research and innovation programme (grant agreement No 805486), and the Belgian Funds for Scientific Research (FRS-FNRS). A. Connolly acknowledges support from NSF Award \#1806923. S. Wissel was supported by NSF CAREER Awards \#1752922 and \#2033500. DZB is 
grateful for support from the U.S. National Science Foundation-EPSCoR (RII Track-2 FEC, award ID 2019597). We express our gratitude to R. Dallier, L. Martin, J-L. Beney and the CODALEMA experiment for providing electronics and hardware to be used in the surface radio stations of RET-CR. Computing resources were provided by the Ohio Supercomputer Center.

\section{References}

[1] ICECube collaboration, First observation of PeV-energy neutrinos with IceCube, Phys. Rev. Lett. 111 (2013) 021103 [1304.5356].

[2] ICECUBE collaboration, Evidence for High-Energy Extraterrestrial Neutrinos at the IceCube Detector, Science 342 (2013) 1242856 [1311 . 5238].

[3] M. Aartsen, M. Ackermann, J. Adams, J.A. Aguilar, M. Ahlers, M. Ahrens et al., Multimessenger observations of a flaring blazar coincident with high-energy neutrino icecube-170922a, Science 361 (2018) eaat1378.

[4] ICECUBE collaboration, Neutrino emission from the direction of the blazar TXS 0506+056 prior to the IceCube-170922A alert, Science 361 (2018) 147.

[5] P. Allison et al., Design and Initial Performance of the Askaryan Radio Array Prototype EeV Neutrino Detector at the South Pole, Astropart. Phys. 35 (2012) 457 [1105 . 2854].

[6] S.R. Klein et al., A radio detector array for cosmic neutrinos on the ross ice shelf, IEEE Transactions on Nuclear Science 60 (2013) 637.

[7] J. Aguilar, P. Allison, J. Beatty, H. Bernhoff, D. Besson, N. Bingefors et al., Design and sensitivity of the radio neutrino observatory in greenland (rno-g), Journal of Instrumentation 16 (2021) P03025.

[8] ANITA collaboration, The Antarctic Impulsive Transient Antenna Ultra-high Energy Neutrino Detector Design, Performance, and Sensitivity for 2006-2007 Balloon Flight, Astropart. Phys. 32 (2009) 10 [0812 . 1920].

[9] GRAND collaboration, The Giant Radio Array for Neutrino Detection (GRAND): Science and Design, 1810.09994.

[10] A. Aab, P. Abreu, M. Aglietta, J.M. Albury, I. Allekotte, A. Almela et al., A search for ultra-high-energy neutrinos from txs 0506+056 using the pierre auger observatory, The Astrophysical Journal 902 (2020) 105.

[11] P. Blackett and C. Lovell, Radio echoes and cosmic ray showers, Proc. Roy. Soc A 177 (1941) 183.

[12] P. Gorham, On the possibility of radar echo detection of ultrahigh-energy cosmic ray induced and neutrino induced extensive air showers, Astropart. Phys. 15 (2001) 177

[hep-ex/0001041]. 
[13] R.U. Abbasi et al., First Upper Limits on the Radar Cross Section of Cosmic-Ray Induced Extensive Air Showers, Astropart. Phys. 87 (2017) 1 [1603.05217].

[14] J. Stasielak et al., Feasibility of radar detection of extensive air showers, Astroparticle Physics 73 (2016) 14.

[15] M. Chiba et al., Reflection of microwave from energy deposit by $x$-ray irradiation in rock salt, SUSYO7 (2007) .

[16] K.D. de Vries, K. Hanson and T. Meures, On the feasibility of RADAR detection of high-energy neutrino-induced showers in ice, Astropart. Phys. 60 (2015) 25 [1312 .4331].

[17] K. de Vries, A. Ó Murchadha, K. Hanson and T. Meures, On the feasibility of the radar detection of high-energy cosmic neutrinos, Proceedings of The 34th International Cosmic Ray Conference - PoS(ICRC2015) 236 (2016) 1168.

[18] K. de Vries et al., Probing the radar scattering cross-section for high-energy particle cascades in ice, Proceedings of Science (ICRC2017) (2017) 1049.

[19] S. Prohira et al., Suggestion of Coherent Radio Reflections from an Electron-Beam Induced Particle Cascade, Phys. Rev. D100 (2019) 072003 [1810. 09914].

[20] S. Prohira et al., Observation of Radar Echoes From High-Energy Particle Cascades, Phys. Rev. Lett. 124 (2020) 091101 [1910. 12830].

[21] S. Prohira and D. Besson, Particle-level model for radar based detection of high-energy neutrino cascades, Nucl. Instrum. Meth. A922 (2019) 161 [1710.02883].

[22] S. Prohira et al., The Radar Echo Telescope for Cosmic Rays: Pathfinder Experiment for a Next-Generation Neutrino Observatory, 2104.00459.

[23] RADAR ECHO TELESCOPE collaboration, Modeling in-ice radio propagation with parabolic equation methods, 2011.05997.

[24] S. Kunwar et al., Design, Construction and Operation of a Low-Power, Autonomous Radio-Frequency Data-Acquisition Station for the TARA Experiment, Nucl. Instrum. Meth. A797 (2015) 110 [1504.00779].

[25] S. Prohira, S. Kunwar, K. Ratzlaff, R. Young and D. Besson, Implementation of a custom time-domain firmware trigger for RADAR-based cosmic ray detection, Nucl. Instrum. Meth. A890 (2018) 126 [1709. 08587]. 


\section{Full Authors List: Radar Echo Telescope Collaboration}

S. Prohira ${ }^{1}$, K.D. de Vries ${ }^{2}$, P. Allison ${ }^{1}$, J. Beatty ${ }^{1}$, D. Besson ${ }^{3,4}$, A. Connolly ${ }^{1}$, P. Dasgupta ${ }^{5}$, C. Deaconu ${ }^{6}$, S. De Kockere ${ }^{2}$, D.

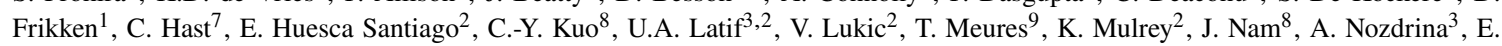
Oberla $^{6}$, J.P. Ralston ${ }^{3}$, C. Sbrocco ${ }^{1}$, R.S. Stanley ${ }^{2}$, J. Torres ${ }^{1}$, S. Toscano ${ }^{5}$, D. Van den Broeck ${ }^{2}$, N. van Eijndhoven ${ }^{2}$, and S. Wissel ${ }^{10,11}$

${ }^{1}$ Department of Physics, Center for Cosmology and AstroParticle Physics (CCAPP), The Ohio State University, Columbus OH, USA

${ }^{2}$ Vrije Universiteit Brussel, Brussel, Belgium

${ }^{3}$ University of Kansas, Lawrence, KS, USA

${ }^{4}$ National Research Nuclear University, Moscow Engineering Physics Institute, Moscow, Russia

${ }^{5}$ Université Libre de Bruxelles, Brussels, Belgium

${ }^{6}$ Enrico Fermi Institute, Kavli Institute for Cosmological Physics,Department of Physics, University of Chicago, Chicago, IL, USA

${ }^{7}$ SLAC National Accelerator Laboratory, Menlo Park, CA, USA

${ }^{8}$ National Taiwan University, Taipei, Taiwan

${ }^{9}$ University of Wisconsin-Madison, Madison, WI, USA

${ }^{10}$ Departments of Physics and Astronomy \& Astrophysics,Institute for Gravitation and the Cosmos, Pennsylvania State University, University Park, PA, USA

${ }^{11}$ California Polytechnic State University, San Luis Obispo CA, USA 